\title{
A quality assessment of Spatial TDR soil moisture measurements in homogenous and heterogeneous media with laboratory experiments
}

\author{
T. Graeff ${ }^{1}$, E. Zehe ${ }^{2}$, S. Schlaeger ${ }^{3}$, M. Morgner ${ }^{3}$, A. Bauer ${ }^{1}$, R. Becker ${ }^{4}$, B. Creutzfeldt ${ }^{5}$, and A. Bronstert ${ }^{1}$ \\ ${ }^{1}$ Institute of Earth and Environmental Sciences, Section Hydrology/Climatology, University of Potsdam, Germany \\ ${ }^{2}$ Institute of Water and Environment, Department of Hydrology and River Basin Management, Technische Universität \\ München, Munich, Germany \\ ${ }^{3}$ SCHLAEGER - mathematical solutions \& engineering, Horn-Bad Meinberg, Germany \\ ${ }^{4}$ Rhine-Waal University of Applied Science, Germany \\ ${ }^{5}$ Deutsches GeoForschungsZentrum GFZ, Section 5.4: Hydrology, Potsdam, Germany
}

Received: 4 December 2009 - Published in Hydrol. Earth Syst. Sci. Discuss.: 14 January 2010

Revised: 27 April 2010 - Accepted: 6 June 2010 - Published: 24 June 2010

\begin{abstract}
Investigation of transient soil moisture profiles yields valuable information of near- surface processes. A recently developed reconstruction algorithm based on the telegraph equation allows the inverse estimation of soil moisture profiles along coated, three rod TDR probes. Laboratory experiments were carried out to prove the results of the inversion and to understand the influence of probe rod deformation and solid objects close to the probe in heterogeneous media. Differences in rod geometry can lead to serious misinterpretations in the soil moisture profile, but have small influence on the average soil moisture along the probe. Solids in the integration volume have almost no effect on average soil moisture, but result in locally slightly decreased moisture values. Inverted profiles obtained in a loamy soil with a clay content of about $16 \%$ were in good agreement with independent measurements.
\end{abstract}

\section{Introduction}

Only a minute amount of global water is stored as soil moisture: with an estimated volume of about $16500 \mathrm{~km}^{3}$, soil moisture represents $0.0012 \%$ and $0.05 \%$ of total and fresh water, respectively (Dingman, 1994). And yet, this tiny hydrological compartment exerts crucial control over interactions between the atmosphere, land surface and groundwater,

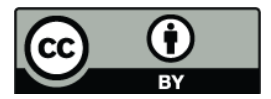

Correspondence to: T. Graeff (graeff@uni-potsdam.de) since soil moisture determines the partitioning of net radiation energy on latent and sensible heat flux and the supply of water for the terrestrial biomass. Furthermore, soil moisture influences plot scale generation of Hortonian and saturated excess overland flow (Chaves et al., 2008; Zehe et al., 2007) and water repellency (Blume et al., 2009), as well as hillslope and catchment scale runoff response to extreme precipitation (e.g. Merz and Bárdossy, 1998; Zehe and Blöschl, 2004).

Spatially and temporally distributed Time Domain Reflectometry (TDR) and Frequency Domain Reflectometry (FDR) measurements are widely used to observe soil moisture dynamics at the plot to hillslope scale (e.g. Starr and Timlin, 2002). Conventional TDR measurements allow estimation of the mean soil moisture and the bulk electrical conductivity of the surrounding media based on the travel time of a reflected electromagnetic wave guided in a waveguide/TDR probe installed in the soil. Excellent reviews are given by Robinson et al. (2003) and Cassiani et al. (2006). Several authors have shown that the shape of the reflected TDR signal, the reflectogram, contains information about the dielectric permittivity $(\varepsilon)$ and thus the soil moisture along the probe (Oswald et al., 2003; Schlaeger, 2005). The retrieval of this detailed information is achieved by inversion or by graphical interpretation of the signal (Moret et al., 2006). Inverse estimation of the soil moisture profile seems to work well for synthetic data sets (Oswald, 2000), homogeneous soils at the lab scale (Becker, 2004; Greco, 2006; Bänninger et al., 2008) or volcanic ash soils with low bulk densities of $\sim 1.0 \mathrm{~g} \mathrm{~cm}^{-3}$ (Greco and Guida, 2008).

Published by Copernicus Publications on behalf of the European Geosciences Union. 


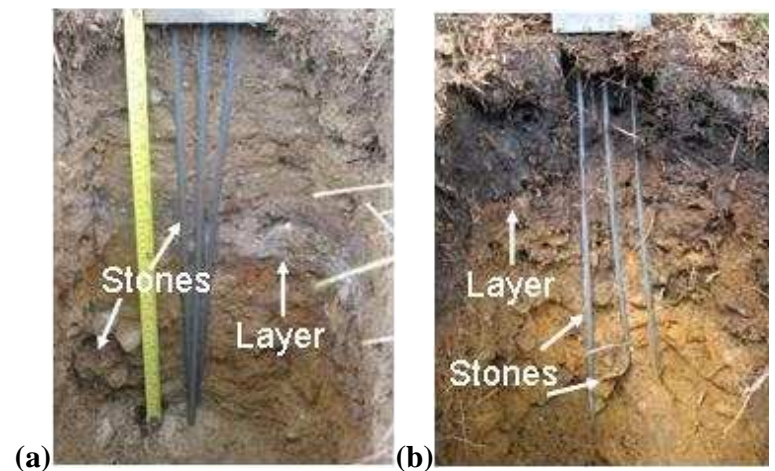

Fig. 1. Typical soil profiles with different horizons and coarse gravel and possible deformations of the TDR wave guides when installed in the soil (a and b).

The essential idea of Spatial TDR is to cluster several wave guides in a small area, operate them by a single sampling TDR and invert the reflectograms to elucidate the evolution of the soil moisture profile. Spatial TDR was originally proposed by Schlaeger (2005) and further tested by Scheuermann et al. (2009) to monitor moisture in sandy dams. The reflectogram of the TDR measurement is influenced by the probe geometry (Bänninger et al., 2008; Spittlehouse, 2000), solids in the sphere of influence (Knight et al., 1997), layered soils (Greco, 2008) or energy dissipation along the probe due to clay and salinity (Jones and Or, 2004; Chen et al., 2007; Kupfer et al., 2007). These different factors may hamper the application of Spatial TDR measurements in real world settings. The use of coated rods protects the TDR signal from energy dissipation (Ferré et al., 1998; Nichol et al., 2002), increasing the signal-to-noise ratio and thus allows the use of longer TDR rods compared to uncoated rods, which is essential for Spatial TDR applications (Dalton and Van Genuchten, 1986). The drawbacks of coated rods are that they are less sensitive to $\varepsilon$, that a coated material needs specific calibration (Ferré et al., 1996), and that the measurement of bulk electrical conductivity is restricted (Moret-Fernández et al., 2009). As the high clay content of the soils in the study area is around $16 \%$, we preferred to use TDR probes with three coated rods. This allows the use of $0.60 \mathrm{~m}$ long probes, which is favourable for the observation of infiltration processes into the subsurface.

In general, it is assumed that the TDR rods are installed parallel, but this is difficult to actually achieve when installing probes in natural soils, especially in the presence of stones, layers, or soil bulk density differences. Figure 1 shows an extreme example with rods converging or diverging with increasing depth for a rather heterogeneous soil located in the Ore Mountains, Saxony, Germany.

The effect of the probe deformation on the reflectogram and the retrieved soil moisture has thus to be studied in detail, because it is essential for the Spatial TDR approach to use long TDR rods. The influence of insulating solids in the sampling volume was theoretically described by Knight et al. (1997), but we are not aware of any study which studied the influence of solids on the reflectogram.

The overall objective of this paper is to shed light on the applicability of Spatial TDR in strongly heterogeneous field soils. Therefore, the questions posed for this research are: 1) How do coated probes of $0.60 \mathrm{~m}$ length react in these soils (heterogeneous, electrical loss), and are these probes indeed better suited for these soils compared to uncoated probes? 2) How does the effect of a) different probe deformations, b) solids (insulators, conductors) in the integration volume and c) high clay content in combination with a bulk density gradient influence the $\varepsilon$ profile, the inverted moisture profiles and the average soil moisture along the probe? Different laboratory experiments were performed to shed light on these topics.

In Sect. 2 we give a review of different inversion techniques. Section 3 provides details on the technological components and discusses potential sources of errors observed in field applications. Section 4 introduces five different laboratory experiments. Section 5 discusses the step from applications in homogenous media or "the technical scale" to reliable applications in heterogeneous field soils, for instance when designing a site-specific calibration of transmission line properties. This step is crucial for hydrology because the relationship between soil moisture dynamics and runoff generation is not well understood yet; this is especially true for heterogeneous soils. These results are furthermore necessary to interpret soil moisture observations obtained with two Spatial TDR clusters installed in the Eastern Ore Mountains, presented in a closely related study by Zehe et al. (2010). That study will introduce the applicability of Spatial TDR in the field scale.

\section{Theoretical background and signal constrained inversion}

\subsection{TDR inversion approaches}

In this section we give an introduction to the inversion methodology and an overview of the different inversion techniques. Generally, it is necessary for the estimation of the soil moisture profiles along the TDR probe to simulate the propagation of the TDR signal in time domain by employing a numerical model (forward problem). This is achieved by simulating the forward and back propagation of the TDR signal along the wave guide and minimizing the differences between observed and simulated signals by using an optimization algorithm which updates the parameter profile along the transmission line. Full wave approaches solve Maxwell's equations within the forward step (Rejiba et al., 2005). The target parameter of the optimization is the profile of the $\varepsilon$ along the wave guide. Other studies have 
proposed simplified approaches based on multi-section transmission lines (Heimovaara et al., 2004) or heterogeneous transmission lines (Greco, 2006); the Spatial TDR approach (Schlaeger, 2005) belongs to the latter category. The wave propagation along the TDR probe is approximated by the telegrapher's equation. The transmission line is conceptualized as a series of bulk electronic components such as resistors, inductors and capacitors. Hence, the target parameter of the optimization is the electrical capacitance profile $(C)$. The Spatial TDR algorithm requires additional material laws that link $C$ - $\varepsilon$-soil moisture and - in the case of TDR probes with coated rods $-C$ and electrical conductance $G$ of the transmission line (compare Sect. 2.3).

\subsection{STDR Signal inversion}

The TDR signal $V_{R}^{o}\left(t, x_{0},\right)$ or reflectogram is a superposition of the input voltage $V_{I}^{o}\left(t, x_{0}\right)$, generated by the TDR device, and partial reflections of the input signal occurring at the junction of the probe and cable as well as at the end of the wave guide. The average $\varepsilon$ along the transmission line is determined by the speed of the electromagnetic wave and can be calculated based on the travel time of the TDR signal. The average $\varepsilon$ can be transformed into the average soil moisture content along the probe by appropriate calibration functions (see Sect. 3.4). The form of the reflectogram between the first and second main reflection at the probe's beginning and end is a finger print of the dielectric profile along the wave guide.

The principle of the Spatial TDR inversion is to estimate the capacitance profile $C(x)$ along the wave guide by means of inverse modelling and transform it into a soil moisture profile $\theta(x)$. As explained above, the forward step of the Spatial TDR algorithm is based on the telegrapher's equation, which describes the propagation of a voltage pulse $V(x, t)$ along the transmission line:

$$
\left(L(x) C(x) \frac{\partial^{2}}{\partial t^{2}}+L(x) G(x) \frac{\partial}{\partial t}+\frac{\partial L(x) / \partial x}{L(x)} \frac{\partial}{\partial t}-\frac{\partial^{2}}{\partial x^{2}}\right) V(x, t)=0 .
$$

Hereby $t$ is time and $x$ the spatial coordinate along the wave guide. The capacitance $C(x)$ and electrical conductance $G(x)$ are both affected by the soil moisture profile $\theta(x)$ along the transmission line. The inductance $L(x)$ is a function of the transmission-line only and piecewise constant for the coaxial cable and moisture probe, as long as the rods are parallel. The spatial derivative of $\mathrm{L}$ in Eq. (1) accounts for the difference between coaxial cable and probe. Compared to the general telegrapher's equation, it is assumed that resistive losses along the probe can be neglected and the electrical resistance $R=0$. All parameter profiles will be given as specific values per unit length. Nichol et al. (2002) have shown that the true electric conductivity $\rho$ cannot be measured with coated probes. Therefore, $G$ is not the real ionic conductance of the soil but an effective value of coating and soil conductivity.
Within the inverse procedure Eq. (1) is numerically solved with appropriate initial and boundary conditions to simulate $V_{R}^{s}\left(t, x_{0} \mid C\right)$ for given parameter profiles $C(x)$ and $G(x)$. Based on the difference between the simulated $V_{R}^{s}\left(t, x_{i} \mid C\right)$ and observed signal $V_{R}^{o}\left(t, x_{i}\right)$ between the first (at $\left.t=0\right)$ and the second main reflection (at $t=T$ ), the transmission line parameters $C(x)$ and $G(x)$ are updated by the conjugate gradient method until the objective function $J(C)$ in Eq. (2) is minimized.

$J(C)=\int_{0}^{T}\left(V_{R}^{s}\left(t, x_{i} \mid C\right)-V_{R}^{o}\left(t, x_{i}\right)\right)^{2} d t$

The high quality of the recorded signal of the TDR100 (Campbell Scientific Inc), which has a time to peak of roughly $200 \mathrm{ps}$, allows inversion at a spatial resolution of $0.01 \mathrm{~m}$ (Oswald et al., 2003; Lin et al., 2005). The solution of Eq. (1) is a profile of $C(x)$ which has to be related to the permittivity profile of the porous medium $\varepsilon(x)$ and finally to the moisture profile $\theta(x)$ (compare next sections). Subsequently, we will refer to the resulting soil moisture profile which is obtained after conversion as the inverted moisture profile. For more details see Schlaeger (2005).

\section{Parameters and potential error sources of spatial TDR-measurements}

\subsection{Technological components and setup of a spatial TDR}

We used a TDR100 by Campbell Scientific Inc. to generate TDR pulses. Coated three-rod probes of type SUSU03 with a length of $0.60 \mathrm{~m}$ developed by Schädel (2006) were used as wave guides. These consist of a stainless steel core of $6 \mathrm{~mm}$ diameter with a $1 \mathrm{~mm}$ thick PVC coating. The distance between the rods is $0.03 \mathrm{~m}$. The rods are screwed into the probe head that is connected to a $50 \Omega$ coaxial cable of type RG213.The probes are connected to an eight channel multiplexer of type SNAPMUX (Becker, 2004) with coaxial cables of type RG213 with an impedance of $50 \Omega$ and a length of $15 \mathrm{~m}$. The TDR 100 is controlled and the data are logged by an ARCOM VIPER 1.2 Industrial-PC with embedded LINUX operating system.

\subsection{Calibration of probe parameters}

The pulse velocity of the TDR signal $v$ is given by $v=2 l / \Delta t$,

where $l$ is the probe length and $\Delta t$ the time difference between the first two main reflections in the reflectogram.

The equation to link $v$ to $\varepsilon$ with $v=c_{0} / \sqrt{\varepsilon}$, with $c_{0}$ as the speed of light, does not apply for coated probes, because here the signal depends on an effective $\varepsilon$ which is composed of the dielectric properties of the coating and of the surrounding medium and would lead to an underestimation of soil 


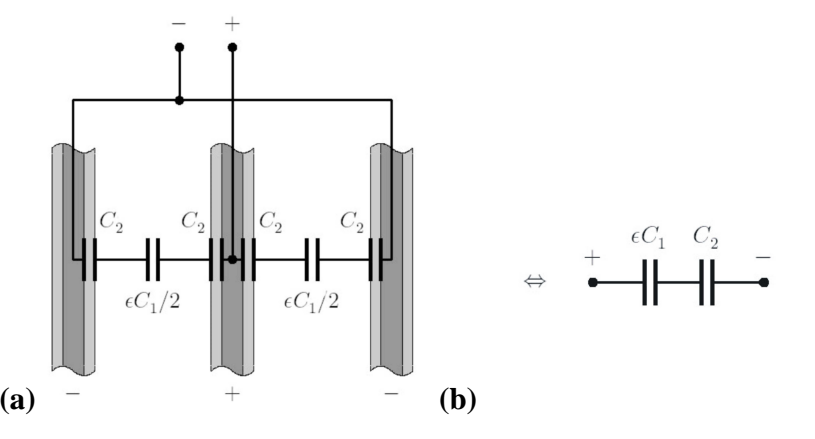

Fig. 2. Total capacitance $C$ of a 3-rod-probe as a function of the soils dielectric permittivitye. (a) segment of three parallel rods encompassed by soil; light grey: PVC coating; dark gray: metallic core; (b) equivalent circuit. $C_{1}, C_{2}$ : constant capacitance parameters determined by the probes geometry (Becker, 2004).

moisture (Ferré et al., 1996). Becker (2004) and Huebner et al. (2005) suggested that the pulse velocity $v(\varepsilon)$ can be best expressed by the constant inductance $L$ of probe and the effective capacitance $C(\varepsilon)$ of the system probe and medium:

$v(\varepsilon)=1 / \sqrt{L \cdot C(\varepsilon)}$.

In a second step $\varepsilon$ of the medium is estimated with a relationship between $C(\varepsilon)$ and $\varepsilon$ in the case of our three rod TDR probe described by a simple circuit model consisting of a series of capacitors, representing the capacitor between the rods filled with the surrounding medium $C_{1}$ and describing the constant capacitance of the coating $C_{2}$ (Fig. 2):

$1 / C(\varepsilon)=1 /\left(\varepsilon \cdot C_{1}\right)+1 / C_{2}$.

According to Eqs. (4) and (5) the probe is characterized by the three parameters $C_{2}, C_{1}$, and $L$, which have to be estimated by calibration measurements. $C_{1}$ and $L$ are affected by the probe geometry, especially the distance of the wave guide rods. For parallel rods, the parameters are assumed to be constant. Becker (2004) found a good correspondence of the relationship between $\varepsilon$ and $C$ derived from full wave numerical simulations of coated three rod probes and the capacitance model shown in Eq. (5). Further laboratory observations corroborated the applicability of this capacitance model to parameterize the relationship between $\varepsilon$ and $C$. Becker (2004) suggested a calibration approach based on measuring TDR pulse velocities $v_{i}=v\left(\varepsilon_{i}\right)$ for two different media with well known dielectric permittivity values $\varepsilon_{1}$ and $\varepsilon_{2}$ (water and air) to determine $C_{2}, C_{1}$, and $L$. Combining Eq. (4) and Eq. (5) for the two media and solving them for $C_{1}$ and $C_{2}$ yields:

$C_{1}=\left(\varepsilon_{2}-\varepsilon_{1}\right) /\left(\varepsilon_{2} \varepsilon_{1}\left(v_{1}^{2}-v_{2}^{2}\right) \cdot L\right.$,

and

$C_{2}=\left(\varepsilon_{2}-\varepsilon_{1}\right) /\left(\left(\varepsilon_{2} v_{2}^{2}-\varepsilon_{1} v_{1}^{2}\right) \cdot L\right)$.
Table 1. Probe parameters estimated based on Eqs. (5) and (9) and absolute errors calculated with Gauss law.

\begin{tabular}{lll}
\hline Parameter & Value & Abs. Error \\
\hline$L\left[\mathrm{nH} \mathrm{m}^{-1}\right]$ & 625.0 & 23.0 \\
$C_{1}\left[\mathrm{pF} \mathrm{m}{ }^{-1}\right]$ & 22.4 & 0.9 \\
$C_{2}\left[\mathrm{pF} \mathrm{m}{ }^{-1}\right]$ & 304.6 & 30.0 \\
\hline
\end{tabular}

Finally $L$ is estimated with the relationship to the $\operatorname{rod}$ impedance:

$Z(\varepsilon)=\sqrt{L / C(\varepsilon)}$.

The jump between the impedance of the probe plus the surrounding medium $Z(\varepsilon)$ and the impedance of the connecting cable $Z_{0}$ causes a partial reflection of the TDR signal at the junction of cable and probe. By measuring the amplitudes of incoming and reflected signal, denoted by $A_{I}$ and $A_{R}$, we obtain the reflection coefficient that is linked to the impedance as follows:

$r(\varepsilon)=A_{R} / A_{I}=\left(Z(\varepsilon)-Z_{0}\right) /\left(Z(\varepsilon)+Z_{0}\right)$,

Substitution of Eq. (4) and Eq. (7) into Eq. (8) and solving for $L$ yields:

$L=(1+r(\varepsilon)) /(1-r(\varepsilon)) \cdot Z_{0} / v(\varepsilon)$.

Based on Eqs. (6) and Eq. (9), the probe parameters $C_{1}, C_{2}$, and $L$ have been derived from TDR reflectograms obtained in de-ionized water $\left(\varepsilon=80\right.$ at $\left.20^{\circ} \mathrm{C}\right)$ and air $(\varepsilon=1)$ with parallel rod geometry for all probes as presented in Table 1. Based on the standard deviation of the dielectric permittivity values we could additionally quantify the relative measurement error to $5 \%$.

\subsection{C-G relation}

As coated rods do not allow direct measurements of the electrical conductivity, we employ an empirical function that relates $C(x)$ to $G(x)$, as proposed by Hakansson (1997), to close our set of equations:

$G(C)=\left\{\begin{array}{lll}G_{\infty} \cdot\left(1-\exp \left(-\left(C-C_{0}\right) / C_{d}\right),\right. & \text { if } & C \geq C_{0}, \\ 0, & \text { if } & 0 \leq C \leq C_{0} .\end{array}\right.$

$G_{\infty}$ is the conductance at saturation, $C_{0}$ is a capacity threshold below which conductance is zero and $C_{d}$ determines how fast $C$ reaches its maximum value. Becker (2004) showed with numerical simulations that Eq. (10) is a suitable model. In general, $C_{d}, G_{\infty}$, and $C_{0}$ have to be determined empirically. In a clay-rich soil one could furthermore expect $G$ to be non-zero for a $C$ smaller than $C_{0}$. The parameter estimation requires manual calibration during the inversion of a known soil moisture profile. 
Table 2. Soil texture (following the United States Department of Agriculture (USDA), 1993 classification), bulk density $\rho_{b}$, saturated soil moisture $\theta_{S}$ and permanent wilting point (PWP) of the dominating Cambisoil at the study area Rehefeld, and experimental glass beads. $\rho$ and $\theta_{S}$ were estimated on $1.00 \mathrm{~m}^{3}$ soil cores with grain density of $2.65 \mathrm{~g} \mathrm{~cm}^{-3}$. PWP is the soil moisture at $160 \mathrm{~m}$ pressure head. The glass beads have a grain size ranging from 0.25 to $0.5 \mathrm{~mm}$ in diameter. Standard deviation is abbreviated as SD.

\begin{tabular}{llllllll}
\hline Soil type & $\begin{array}{l}\text { Sand } \\
{[\%]}\end{array}$ & $\begin{array}{l}\text { Silt } \\
{[\%]}\end{array}$ & $\begin{array}{l}\text { Clay } \\
{[\%]}\end{array}$ & $\begin{array}{l}\rho_{b} \\
{\left[\mathrm{~g} \mathrm{~cm}^{-3}\right]}\end{array}$ & $\begin{array}{l}\mathrm{SD}_{\rho_{b}} \\
{\left[\mathrm{~g} \mathrm{~cm}^{-3}\right]}\end{array}$ & $\begin{array}{l}\theta_{S} \\
{\left[\mathrm{~m}^{3} \mathrm{~m}^{-3}\right]}\end{array}$ & $\begin{array}{l}\mathrm{PWP} \\
{\left[\mathrm{m}^{3} \mathrm{~m}^{-3}\right]}\end{array}$ \\
\hline $\begin{array}{l}\text { Cambisoil } \\
\text { Rehefeld }\end{array}$ & 52 & 32 & 16 & 1.15 & 0.11 & 0.56 & 0.08 \\
Glass beads & 100 & - & - & 1.50 & 0.05 & 0.38 & 0.03 \\
\hline
\end{tabular}

\section{$3.4 \varepsilon$-soil moisture relation}

Different models linking $\varepsilon$ and soil moisture are reviewed by Cassiani et al. (2006) and Lesmes and Friedman (2005). Because of different geochemical and geophysical properties it is difficult to find a universal petro-physical relationship for the unsaturated zone (Paasche et al., 2006). In this study, the soil moisture from $\varepsilon$ is calculated using the empirical relation proposed by Topp et al. (1980) for sand and glass beads. The $\varepsilon$-soil moisture relation was developed using 11 undisturbed soil samples in a plastic core cylinder (diameter $=0.057 \mathrm{~m}$, length $0.10 \mathrm{~m}$ ) from different horizons of the study area. In the laboratory, samples were saturated and in each sample a 3-rod $0.075 \mathrm{~m}$ long TDR probe (CS640-L connected to a TDR100, both Campbell Scientific Inc.) were inserted. The samples were slowly dried. Once or twice a day the soil moisture was estimated by gravimetric method and the dielectric permittivity by TDR measurements. Different approaches to link $\varepsilon$ and soil moisture were tested (Alharthi and Land, 1987; Roth et al., 1992; Malicki et al., 1996; Friedman, 1997). For the soils in the study area, the linear relationship between the refractive index and soil moisture (Herkelrath et al., 1991) was found to be most suitable:

$\theta=a+b \cdot \sqrt{\varepsilon}$.

The parameters $a$ and $b$ are fitting parameters.

\subsection{Probe deformations during installation}

We investigated whether Spatial TDR clusters allows assessment of distributed soil moisture profiles under natural conditions in the headwater of the Weißeritz catchment close to the village of Rehefeld in Saxony, Germany. Soils are mainly Cambisols in periglacial weathering covers. In the summer of 2006, we installed two Spatial TDR clusters at two hillslopes close to the village Rehefeld. Table 2 shows the mean soil characteristics determined from 20 undisturbed soil samples extracted in profiles excavated up to a depth of $0.70 \mathrm{~m}$ close to one of the clusters. Additional details on the project context, the spacing of TDR probes and the catchment are discussed in Zehe et al. (2010). The importance for the present study is that the installation of the $0.60 \mathrm{~m}$ long Spatial
TDR probe at this field site was a challenging task, due to the large amount of gravel of up to $0.4 \mathrm{~kg} \mathrm{~kg}^{-1}$ (Landesamt für Umwelt, Landwirtschaft und Geologie, 2006), the increasing density with depth and the heterogeneity of the soils. Similar problems were documented by Spittlehouse (2000). We used a steel template with three holes set at the right distance from each other as well as a power drill with a $0.60 \mathrm{~m}$ long auger. Several attempts (on average about two) were necessary to drill three holes with the appropriate distance and depth due to gravel blocking. Nonetheless, we had difficulties in ensuring that the rods of the probes were parallel. Figure 1 illustrates typical deformations of the probes; the rods converge towards the end (Fig. 1a) or diverge with increasing depth (Fig. 1b). As the theory of the inversion assumes parallel geometry of the rods, these deformations will likely cause errors in the estimated soil moisture profiles, because $C_{1}$ and $L$ cannot be assumed as constant over the profile. In section 3.2, we describe the experimental setup to investigate the influence of simple rod deformations on the inversion.

\subsection{Gravel and stones}

The pulse velocity measured with TDR is related to the average volumetric soil moisture. This can, as suggested by Topp and Davis (1982), lead to misinterpretations when abrupt water content changes along the transmission lines are present. Knight et al. (1997) theoretically discussed the influence of "gaps" in the integration volume which were filled with materials with either a lower than average or higher than average permittivity. They found materials with lower than average permittivity to have stronger impacts on TDR measurements. From a soil physical view, coarse gravel and stones in the integration volume of the TDR probe reduce the total volume of the pore space $\Phi$ at that depth.

\section{Laboratory experiments to quantify error sources}

In this section we present the setup of our five different laboratory experiments and present the results. In experiment 1 to 4 , we used glass beads with a grain size of $0.25-0.5 \mathrm{~mm}$ diameter to ensure that our performance test took place in a medium with homogeneous pore space. In experiment 5 , we 
Table 3. Parameter sets characterizing the $C-G$ relations for inversion of the reflectograms into soil moisture profiles both for glass beads and soils.

\begin{tabular}{|c|c|c|c|c|}
\hline Exp. & Name & $\begin{array}{r}G_{\infty} \\
{\left[\mathrm{mS} \mathrm{m}^{-1}\right]}\end{array}$ & $\begin{array}{r}C_{0} \\
{\left[\mathrm{pF} \mathrm{m}^{-1}\right]}\end{array}$ & $\begin{array}{r}C_{d} \\
{\left[\mathrm{pF} \mathrm{m}^{-1}\right]}\end{array}$ \\
\hline 1 & Glass beads & 1.5 & 50 & 18 \\
\hline 2 & Rehefeld soil & 2.0 & 50 & 18 \\
\hline
\end{tabular}

(a)
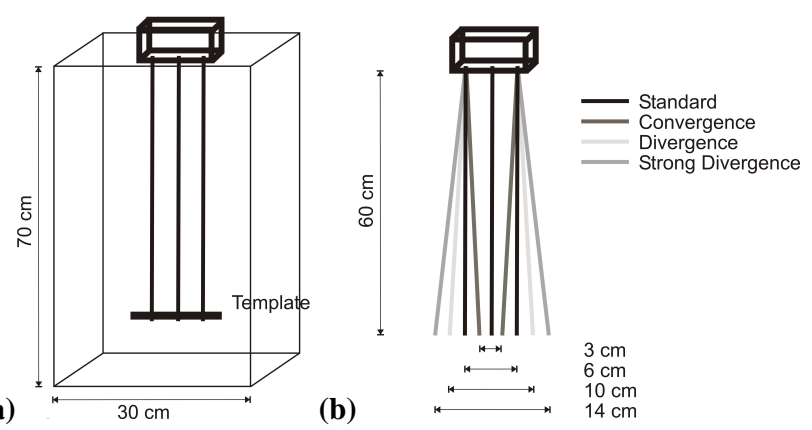

Fig. 3. Sketch of the plastic box with installed SUSU03 and position of the wooden template (a), and sketch of the four different probe geometries (b).

used disturbed soil from the field as described in Table 2 to test the method within a heterogeneous medium.

\subsection{C-G relation, $\varepsilon$-soil moisture relation and constrain- ing of inverted moisture profiles}

We estimated the three parameters $C_{d}, G_{\infty}$, and $C_{0}$ of Eq. (10) within experiments 2 and 5. The results are listed in Table 3. The parameters of Eq. (11) $a$ and $b$ were estimated at -0.2291 and 0.1324 , respectively. The coefficient of determination $R^{2}$ was 0.9837 .

The last crucial step to ensure that the inverted soil moisture profiles complied with soil physics is to constrain the inversion by a physical range. The upper end is defined by soil saturated water content $\left(\theta_{S}\right)$ and the lower end by the permanent wilting point (PWP). This can easily be achieved by using the inverse $\varepsilon$ to $\theta$ and the inverse $C$ to $\varepsilon$ relationships to obtain upper and lower limits for $C$. The parameters for the different soil substrates are listed in Table 2.

\subsection{Experiment 1: effect of uncoated and coated probes on the reflectogram in field soils}

We compared two SUSU03 probes, one of each with and without coating. Experiments were accomplished in a plastic box with a height of $0.70 \mathrm{~m}$ and edge length of $0.30 \mathrm{~m}$ by $0.30 \mathrm{~m}$ (Fig. 3a). Both probes were installed in the middle of the box and we placed a wooden template at $0.59 \mathrm{~m}$ depth to secure ideal probe geometry (Fig. 3a). The box was care-

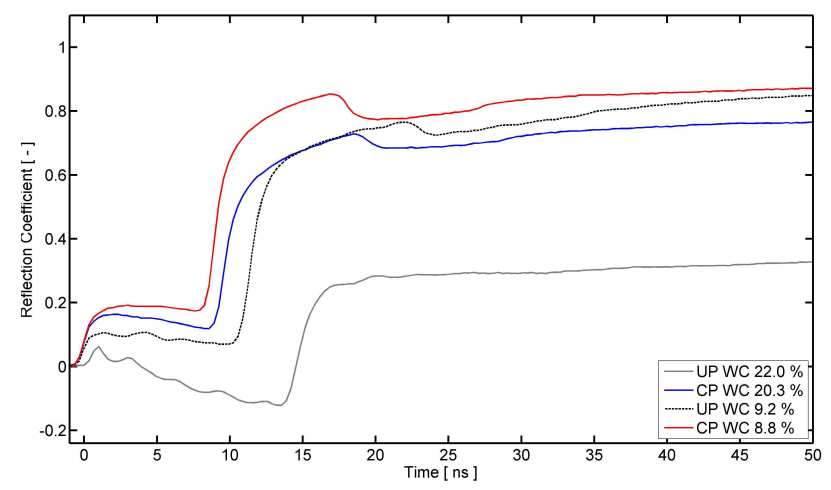

Fig. 4. Reflectograms obtained with coated (CP) and uncoated (UP) SUSU03 probes with $0.60 \mathrm{~m}$ rods at two different soil moistures, measured in an experimental box with glass beads (Table 2). Bulk electrical conductivity was $1.0 \times 10^{-2} \mathrm{dS} \mathrm{m}^{-1}$ for the dry case and $6.7 \times 10^{-2} \mathrm{dS} \mathrm{m}^{-1}$ for the wet case.

fully filled with glass beads that were moderately compacted to ensure good contact between soil and TDR probes (Table 2). The experiment was conducted at two different wetness conditions, namely $0.08-0.09 \mathrm{~m}^{3} \mathrm{~m}^{-3}$ (dry) and $0.20-$ $0.21 \mathrm{~m}^{3} \mathrm{~m}^{-3}$ (wet).

Figure 4 shows the reflectograms of a coated and an uncoated rod probe at two different soil moistures in glass beads. In both cases the travel time of the coated probe is smaller compared to the uncoated which is an effect of the isolating PVC coating. Estimated bulk electrical conductivity based on the method suggested by Huisman et al. (2008) and Lin et al. (2007) yielded values of $1.0 \times 10^{-2} \mathrm{dS} \mathrm{m}^{-1}$ and $6.7 \times 10^{-2} \mathrm{dS} \mathrm{m}^{-1}$ for the dry and wet case, respectively. For the case of uncoated rods, even a low electrical conductivity has already a strong influence on the shape of the reflectogram. Especially in the wet case, there is a strong attenuation between 2 and $14 \mathrm{~ns}$ that is much less pronounced for the coated rods. Despite the low value of bulk electrical conductivity, its influence on the uncoated probe is significantly strong. We thus may state that coated rods will deliver the more reliable reflectograms and should be used in these soils when using a probe length of $0.60 \mathrm{~m}$.

\subsection{Experiment 2: performance in homogeneous media during transient conditions}

Figure 5 shows the experiment setup. The experiment was conducted in a $1 \mathrm{~m}$ high and $0.15 \mathrm{~m}$ wide PVC tube. Glass beads were filled into the tube and compacted, resulting in a bulk density of $1.51 \mathrm{~g} \mathrm{~m}^{-3}$ and a saturated water content of $0.38 \mathrm{~m}^{3} \mathrm{~m}^{-3}$ (Table 2). Two T-pieces with a diameter of $0.15 \mathrm{~m}$ and a length of $0.09 \mathrm{~m}$ in the tube allowed for the installation of THETA probes (THETA, Delta-T-Devices) with a shaft length of $0.11 \mathrm{~m}$, rod length of $0.06 \mathrm{~m}$ and a diameter of $0.04 \mathrm{~m}$. One probe of type SUSU03 was installed in the centre of the tube with rods pointing from the upper 
Table 4. Difference of inverted soil moisture to point measurements with 1 THETA probes as absolute error (AE) of the soil moisture in $\mathrm{m}^{3} \mathrm{~m}^{-3}$; goodness of fit criteria calculated from observed and reconstructed reflectograms: root mean square error (RMSQ), mean error (ME), standard deviation of error (STDE), Nash-Sutcliffe efficiency (NSE) and the objective function (Eq. 2).

\begin{tabular}{llllll}
\hline Time $[d]$ & 0 & 1.5 & $2.5 \mathrm{r}$ & 3.5 & 4.5 \\
\hline AE $0.30 \mathrm{~m}$ & -0.022 & -0.032 & 0.022 & -0.002 & -0.003 \\
AE $0.55 \mathrm{~m}$ & 0.003 & 0.000 & 0.010 & -0.029 & 0.019 \\
RMSQ & $1.50 \times 10^{-3}$ & $4.52 \times 10^{-3}$ & $6.18 \times 10^{-3}$ & $8.05 \times 10^{-3}$ & $6.65 \times 10^{-3}$ \\
ME & $-1.02 \times 10^{-3}$ & $-3.06 \times 10^{-3}$ & $-4.60 \times 10^{-3}$ & $-5.95 \times 10^{-3}$ & $-4.51 \times 10^{-3}$ \\
STDE & $1.10 \times 10^{-3}$ & $3.35 \times 10^{-3}$ & $4.02 \times 10^{-3}$ & $5.06 \times 10^{-3}$ & $4.81 \times 10^{-3}$ \\
NSE & 0.995 & 0.994 & 0.992 & 0.990 & 0.993 \\
Objective & $3.35 \times 10^{-14}$ & $3.06 \times 10^{-13}$ & $5.69 \times 10^{-13}$ & $9.63 \times 10^{-13}$ & $6.55 \times 10^{-13}$ \\
Function & & & & & \\
\hline
\end{tabular}

edge of the tube to the bottom and the narrow side of the probe faced to the T-pieces. Independent soil moisture measurements were obtained with two THETA probes placed at a depth of $0.30 \mathrm{~m}$ and $0.55 \mathrm{~m}$, which work in the FDR domain with a measurement error of $\pm 0.01 \mathrm{~m}^{3} \mathrm{~m}^{-3}$ (Gaskin and Miller, 1996). The THETA probes reach $0.03 \mathrm{~m}$ into the centre tube. The rods of the THETA probes have a distance of $0.04 \mathrm{~m}$ to the rods of the SUSU03 to avoid interaction of the measured signal. The sample area of a coated probe is of low range (Ferré et al., 1998) outside the rods and the additionally reflectograms are proven on the influence of the THETA probes. We started the experiments with a tube that was fully saturated with de-ionized water and the soil moisture was reduced by sucking off $250 \mathrm{ml}$ of water at the bottom of the tube every $6 \mathrm{~h}$. Soil moisture profiles were inverted at the different moisture conditions and compared to the THETA probes.

Figure 6 presents inverted moisture profiles in comparison to the THETA probe obtained during the experiment. For the inversion the parameter set "exp. 1" in Table 3 was used for the inversion. The profile data were aggregated to $0.05 \mathrm{~m}$ for a better comparability. Table 4 shows the absolute error of the inverted profiles to the THETA probes and goodness of fit criteria for the inversion calculated from observed and reconstructed reflectograms. Both data sets are generally in good agreement, except for days 1.5 and 2.5. The higher values of the THETA probe measurements may result from effects of the T-pieces, which may have retained higher water content during drainage. Finally, it is important to stress that even a glass bead medium is not perfectly homogeneous. The reader should note the small variations in the moisture profile at the beginning of the experiment, which reflect small differences in saturated water content.

\subsection{Experiment 3: effect of probe deformations}

The effect of probe deformation on the estimated soil moisture profile and the mean soil moisture were studied by deforming the two outer rods under controlled conditions with

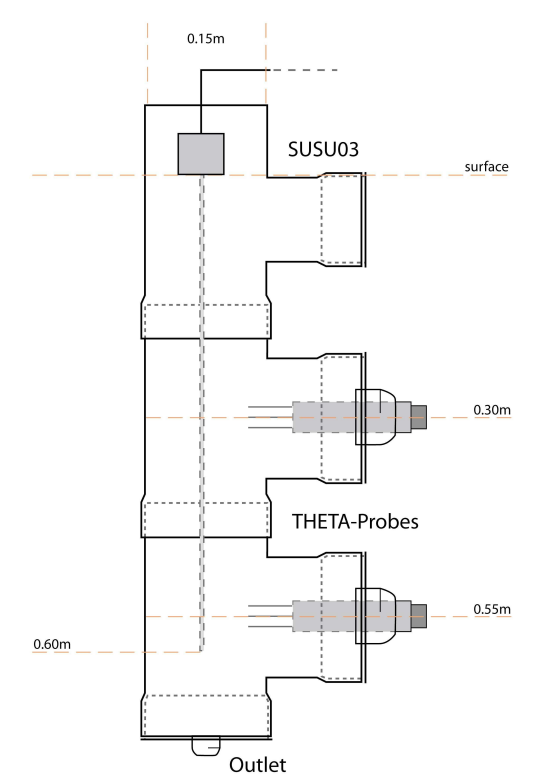

Fig. 5. Sketch of the setup of experiment 2. A SUSU03 probe and two Theta probes were installed in PVC tubes and the tubes were filled with glass beads. At the bottom of the tube, an outlet permits the controlled and stepwise drainage of water in the tube.

the assumption of a parameter set up for a correctly parallel installed probe. Here we studied four different cases: parallel rods, converged rods, diverged rods and strongly diverged rods (Fig. 3b and Table 5). The experiment was conducted with glass beads in a plastic box and a template to ensure the probe deformation (Fig. 3a). The experiment was conducted at three soil moisture levels: $0.04-0.05 \mathrm{~m}^{3} \mathrm{~m}^{-3}$, $0.07-0.09 \mathrm{~m}^{3} \mathrm{~m}^{-3}$, and $0.20-0.23 \mathrm{~m}^{3} \mathrm{~m}^{-3}$. The values were cross-checked with THETA probe measurements along the experimental box. A TDR measurement was performed and inverted into a soil moisture profile. The procedure was repeated for all selected deformations. Establishing a homogeneous soil moisture profile during a single experiment was rather difficult and could only be achieved approximately. 
Table 5. Mean soil moisture observed with different probe deformations at soil moisture of approximately $0.04 \mathrm{~m}^{3} \mathrm{~m}^{-3}, 0.08 \mathrm{~m}^{3} \mathrm{~m}^{-3}$ and $0.20 \mathrm{~m}^{3} \mathrm{~m}^{-3}$ estimated with SUSU03 and THETA probes. Standard deviation is abbreviated as SD.

\begin{tabular}{lllllll}
\hline $\begin{array}{l}\text { Type of } \\
\text { Deformation }\end{array}$ & Convergence & Standard & Divergence & $\begin{array}{l}\text { Strong } \\
\text { Divergence }\end{array}$ & $\begin{array}{l}\text { Mean THETA } \\
\text { probes }\end{array}$ & $\begin{array}{l}\text { SD THETA } \\
\text { probes }\end{array}$ \\
\hline $\begin{array}{l}\text { Distance between } \\
\text { the outer rods [m] }\end{array}$ & 0.03 & 0.06 & 0.10 & 0.14 & - & - \\
$0.04 \mathrm{~m}^{3} \mathrm{~m}^{-3}$ & 0.036 & 0.038 & 0.039 & 0.039 & 0.040 & 0.005 \\
$0.08 \mathrm{~m}^{3} \mathrm{~m}^{-3}$ & 0.077 & 0.081 & 0.079 & 0.073 & 0.080 & 0.010 \\
$0.21 \mathrm{~m}^{3} \mathrm{~m}^{-3}$ & 0.207 & 0.199 & 0.200 & 0.195 & 0.200 & 0.020 \\
\hline
\end{tabular}

Table 6. Amplitude coefficient (CA) for different probe deformations at soil moisture of approximately $0.04 \mathrm{~m}^{3} \mathrm{~m}^{-3}, 0.08 \mathrm{~m}^{3} \mathrm{~m}^{-3}$ and $0.20 \mathrm{~m}^{3} \mathrm{~m}^{-3}$.

\begin{tabular}{ccccc}
\hline $\begin{array}{c}\text { Type of } \\
\text { Deformation }\end{array}$ & Convergence & Standard & Divergence & $\begin{array}{c}\text { Strong } \\
\text { Divergence }\end{array}$ \\
\hline $\begin{array}{c}\text { Distance between } \\
\text { the outer rods [m] }\end{array}$ & 0.03 & 0.06 & 0.10 & 0.14 \\
$0.04 \mathrm{~m}^{3} \mathrm{~m}^{-3}$ & 0.11 & -0.33 & -0.58 & -0.75 \\
$0.08 \mathrm{~m}^{3} \mathrm{~m}^{-3}$ & 0.22 & -0.20 & -0.66 & -0.80 \\
$0.21 \mathrm{~m}^{3} \mathrm{~m}^{-3}$ & 0.46 & -0.25 & -0.58 & -1.31 \\
\hline
\end{tabular}

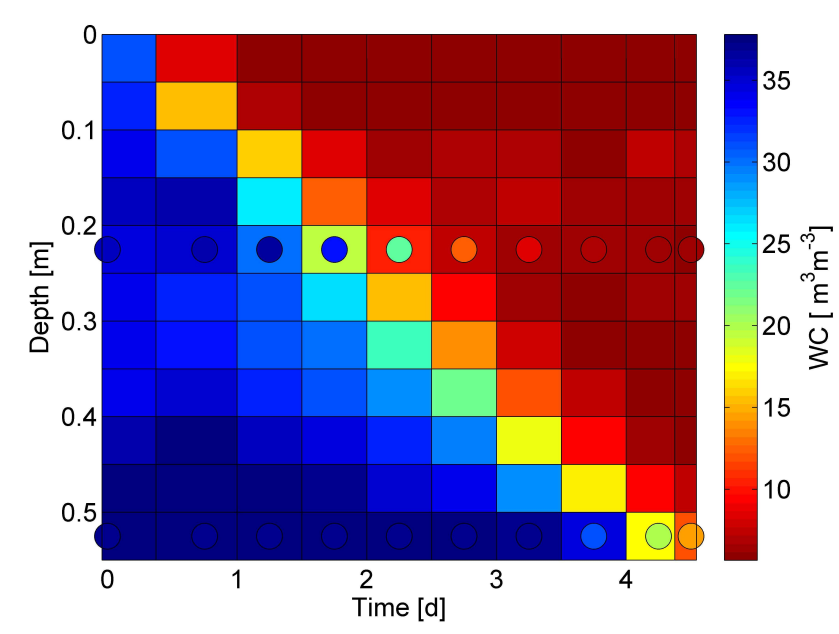

Fig. 6. Comparison of inverted soil moisture profiles obtained within glass beads with independent soil moisture measurements by means of THETA probes (marked with circles). The colour coding is the same for both data sets.

Figure 7 presents the reflectograms and the inverted soil moisture profiles at different average soil moistures of $0.05 \mathrm{~m}^{3} \mathrm{~m}^{-3}$ (Fig. 7a and d), $0.08 \mathrm{~m}^{3} \mathrm{~m}^{-3}$ (Fig. $7 \mathrm{~b}$ and e) and $0.20 \mathrm{~m}^{3} \mathrm{~m}^{-3}$ (Fig. 7c and f) for the four different rod geometries shown in Fig. 3b. During inversion we used the parame- ter set for glass beads (Table 3 ). It has to be noted that due to installation and de-installation of the probe, which required refilling of the box, the soil moisture and bulk density profiles varied slightly between different experiments (Table 5 and Fig. 7a-c) when comparing the different geometries.

A decreasing distance between the wave guide rods means an increasing capacitance of the transmission line. Hence, the probe parameters $C_{1}$ and $L$ should vary along the transmission line. However, they are currently assumed to be constant, because we are studying the effect of rod deformation on the retrieved soil moisture profile by the supposition of a parallel geometry on de facto different geometries. For convergent rods, the average soil moisture was measured correctly compared to the measurement with parallel rods. However, the soil moisture along the rod increases with depth in all three cases, implying an underestimation of soil moisture in the upper half and an overestimation of soil moisture in the lower half. This becomes especially apparent for intermediate conditions and wet conditions.

For the case of divergent rods, the apparent soil moisture profile is just flipped in comparison to the convergent case. Thus, in the upper half we observe an overestimation and in the lower part an underestimation of the soil moisture. It is important to note that the average soil moisture calculated from the travel time with constant parameters is in most cases within the error range almost unaffected by deformations of the probe (Table 5). The experiments were also repeated in 

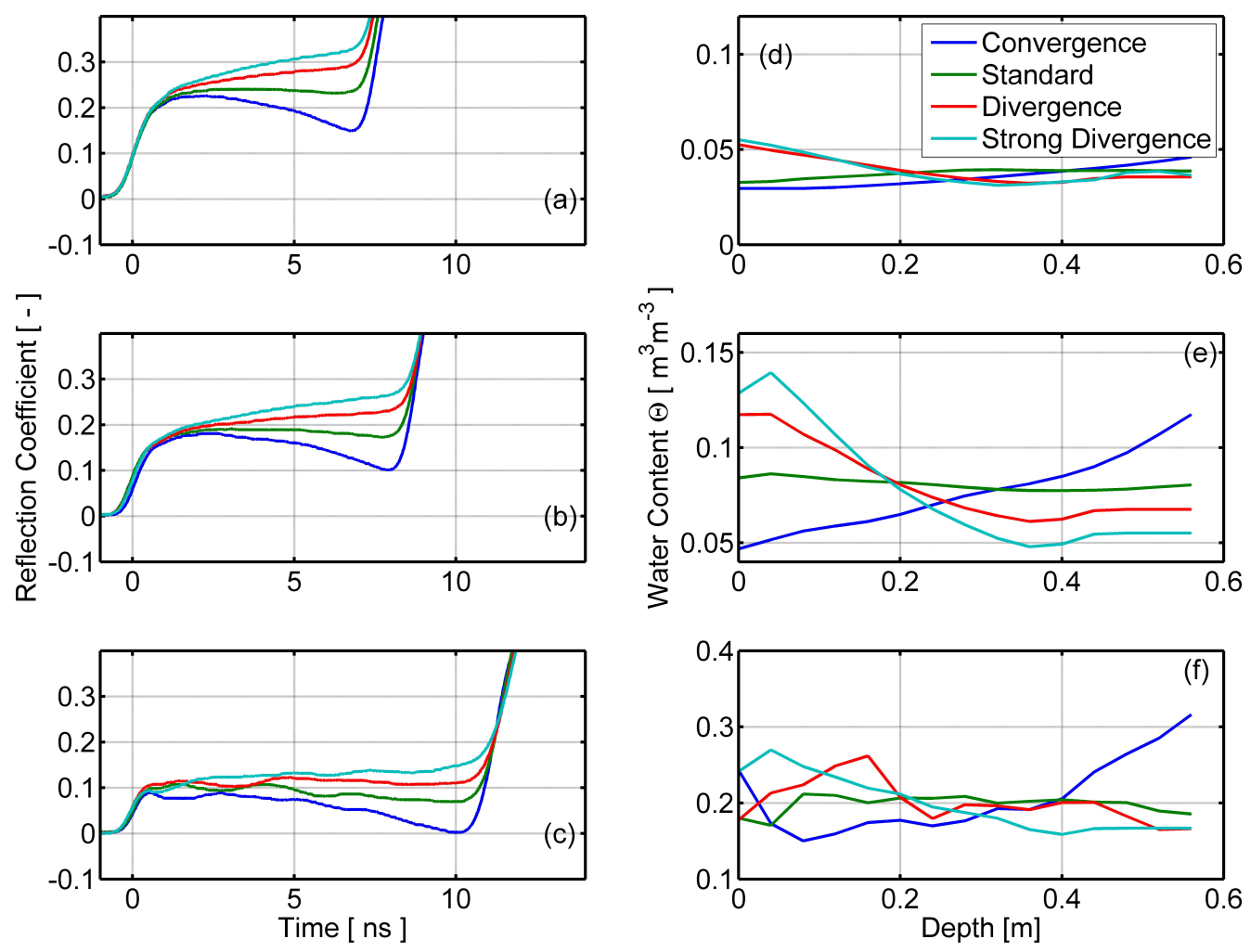

Fig. 7. Reflectograms and inverted soil moisture profiles obtained with different probe deformations at a soil moisture of approximately $0.04 \mathrm{~m}^{3} \mathrm{~m}^{-3}$ (a) and (d); $0.08 \mathrm{~m}^{3} \mathrm{~m}^{-3}$ (b) and (e); and $0.20 \mathrm{~m}^{3} \mathrm{~m}^{-3}$ (c) and (f). Standard denotes according to Fig. 3 ideal geometry, Convergence means convergent rods with increasing depth, Divergence and Strong Divergence values signifies a divergent probe as described in Fig. 3.

coarse sand of 0.06 to $0.60 \mathrm{~mm}$ grain size, with similar results (not shown). Thus, we state that unknown changes in probe geometry will lead to a systematic bias in inverted soil moisture profiles, but will leave the average values largely unchanged in case of the used SUSU03 probe.

As a first step, we tested a simple quality measure to identify probe deformations by introducing the coefficient of amplitude CA, defined as:

$\mathrm{CA}=\left(V_{\max 1}-V_{\min }\right) / V_{\max 1}$

where $V_{\max 1}=$ maximum voltage of the first reflection and $V_{\min }$ is the inflection point before the second reflection in the reflectogram. The corresponding values for the deformation cases are listed in Table 6. In the convergent case, CA has positive values and is negative in the standard and divergent case. With increasing divergence $\mathrm{CA}$ values become larger. This is consistent with the theory of a plate capacitor, as an increasing distance between the rods corresponds to a decreasing conductance. The amplitude at the end of the reflectogram will thus increase, which yields a negative value for $\mathrm{CA}$. In the convergent case the conductance increases at the end of the probe, which means a small amplitude and thus a positive CA. However, in the case of layered soils, where the lower part can be systematically drier/wetter than the upper soil, or case of gradients in salt, clay or organic content identification of probe deformations using Eq. (12) is not that straight forward.

\subsection{Experiment 4: effect of solid objects in the integration volume}

The purpose of this experiment was to study the influence of different solids on the reflectogram and the estimated soil moisture profile. A coated SUSU03 probe was installed in the same box used in experiment 1 (Fig. 3a); ideal parallel geometry was ensured by installing a wooden template at a depth of $0.59 \mathrm{~m}$. An iron block (a conductor), a dry and a saturated piece of wood (insulator), a PVC block (insulator) and a brick or a boulder from the study area, all with a volume of approximately 1.51 , were placed close to the probe at a depth of $0.30 \mathrm{~m}$. The box was filled with glass beads (Table 2). TDR measurements were performed at three different soil moisture levels: $0.04 \mathrm{~m}^{3} \mathrm{~m}^{-3}$ (dry case), $0.16 \mathrm{~m}^{3} \mathrm{~m}^{-3}$ (intermediate case) and $0.30 \mathrm{~m}^{3} \mathrm{~m}^{-3}$ (wet case).

Figure 8 presents the reflectograms as well as the inverted soil moisture profiles for the brick, the iron block, the dry and saturated wood, and the boulder block. Table 7 lists 

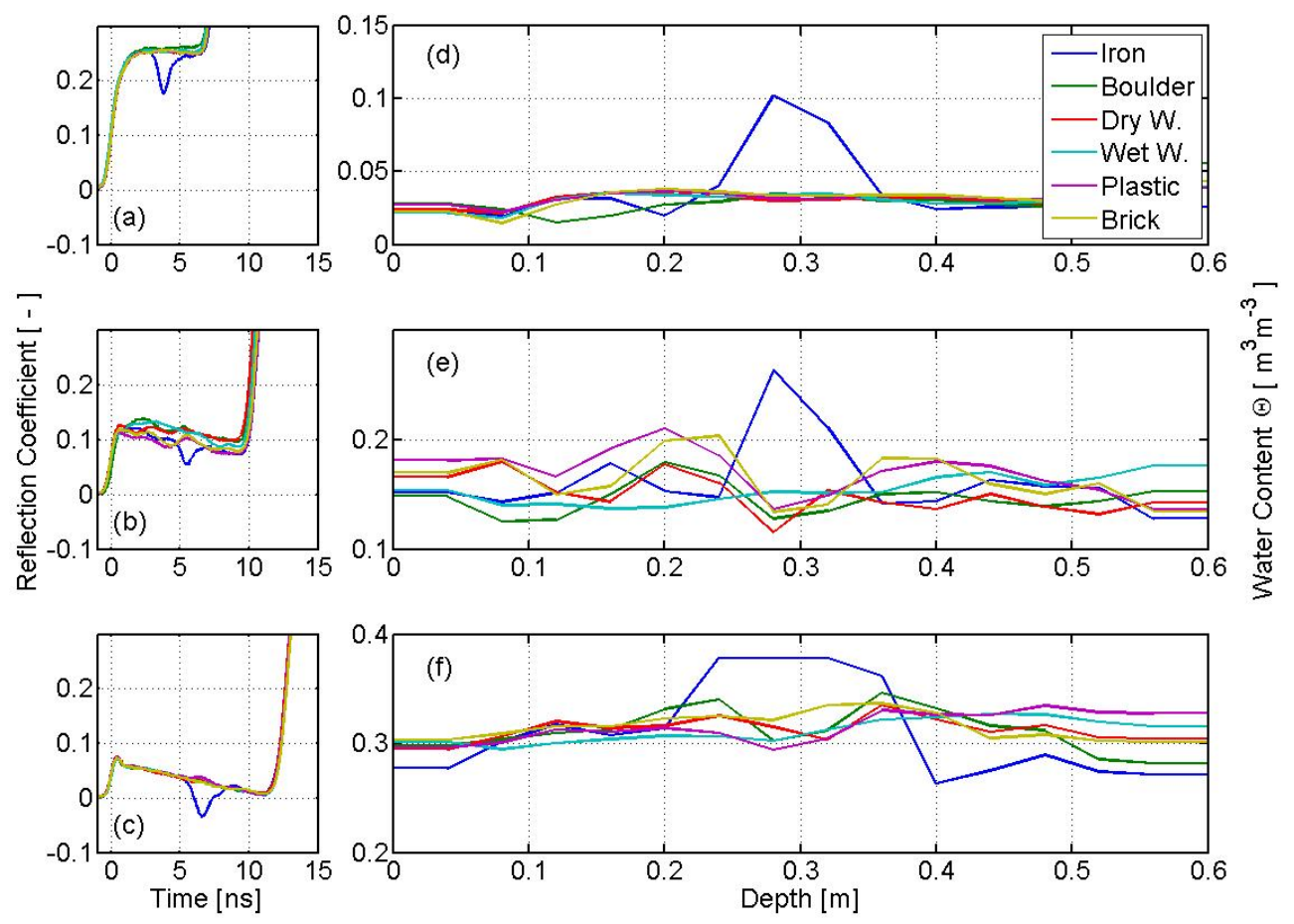

Fig. 8. Reflectograms and inverted soil moisture profiles with an iron block, dry and wet wood, PVC block, brick and boulder with a volume of approximately 1.51 at a depth of $0.30 \mathrm{~m}$. All probes were measured with ideal geometry. The mean soil moisture is about $0.04 \mathrm{~m}^{3} \mathrm{~m}^{-3}$, ((a) and (d), dry case) $0.16 \mathrm{~m}^{3} \mathrm{~m}^{-3}\left(\mathbf{( b )}\right.$ and (e), intermediate case) and $0.30 \mathrm{~m}^{3} \mathrm{~m}^{-3}($ (c) and (f), wet case). Wood is abbreviated to W.

Table 7. Mean soil moisture observed with THETA probes and estimated with the different objects and the soil moisture in the area of the object.

\begin{tabular}{|c|c|c|c|c|c|c|c|}
\hline \multicolumn{8}{|c|}{ Soil moisture between $0.28-0.33 \mathrm{~m}$ depth $\left[\mathrm{m}^{3} \mathrm{~m}^{-3}\right]$} \\
\hline$t$ & $\begin{array}{l}\text { Mean } \\
\text { THETA } \\
\text { probe }\end{array}$ & Iron & Boulder & Dry Wood & Sat.Wood & Plastic & Brick \\
\hline dry & 0.04 & 0.094 & 0.034 & 0.031 & 0.034 & 0.031 & 0.034 \\
\hline intermediate & 0.16 & 0.240 & 0.131 & 0.135 & 0.152 & 0.142 & 0.140 \\
\hline wet & 0.30 & 0.378 & 0.306 & 0.308 & 0.307 & 0.299 & 0.328 \\
\hline \multicolumn{8}{|c|}{ mean soil moisture SUSU03 } \\
\hline dry & 0.04 & 0.037 & 0.034 & 0.034 & 0.033 & 0.034 & 0.035 \\
\hline intermediate & 0.16 & 0.160 & 0.147 & 0.147 & 0.154 & 0.166 & 0.161 \\
\hline wet & 0.30 & 0.310 & 0.313 & 0.313 & 0.312 & 0.315 & 0.316 \\
\hline
\end{tabular}

the soil moisture observed with THETA probe measurements along the profile, the mean soil moisture estimated with the SUSU03 and the soil moisture in the area of the object. During inversion, parameter set "exp. 2" (Table 3) was used to characterize the transmission line. Similar to experiment 3 it has to be noted that due to installation and de-installation of the probe, soil moisture and bulk density profiles varied slightly between different experiments. This leads to a nonconstant profile in the inverted profiles, especially in the intermediate and wet case (Fig. 8e and f).
As the iron block is an ideal conductor, the electric conductivity is strongly increased at a depth of $0.30 \mathrm{~m}$. Consequently, soil moisture appears to be higher at that depth in the soil moisture profile, which is indicated in the reflectogram by the pronounced decrease in the amplitude at $5 \mathrm{~ns}$ in Fig. 8c. The inversion yielded a soil moisture value of $0.37 \mathrm{~m}^{3} \mathrm{~m}^{-3}$ at $0.30 \mathrm{~m}$, whereas the true soil moisture was approximately $0.30 \mathrm{~m}^{3} \mathrm{~m}^{-3}$. The other objects, with the exception of the wet piece of wood, show up as a slightly drier region in the reflectogram, marked by the small increase in 


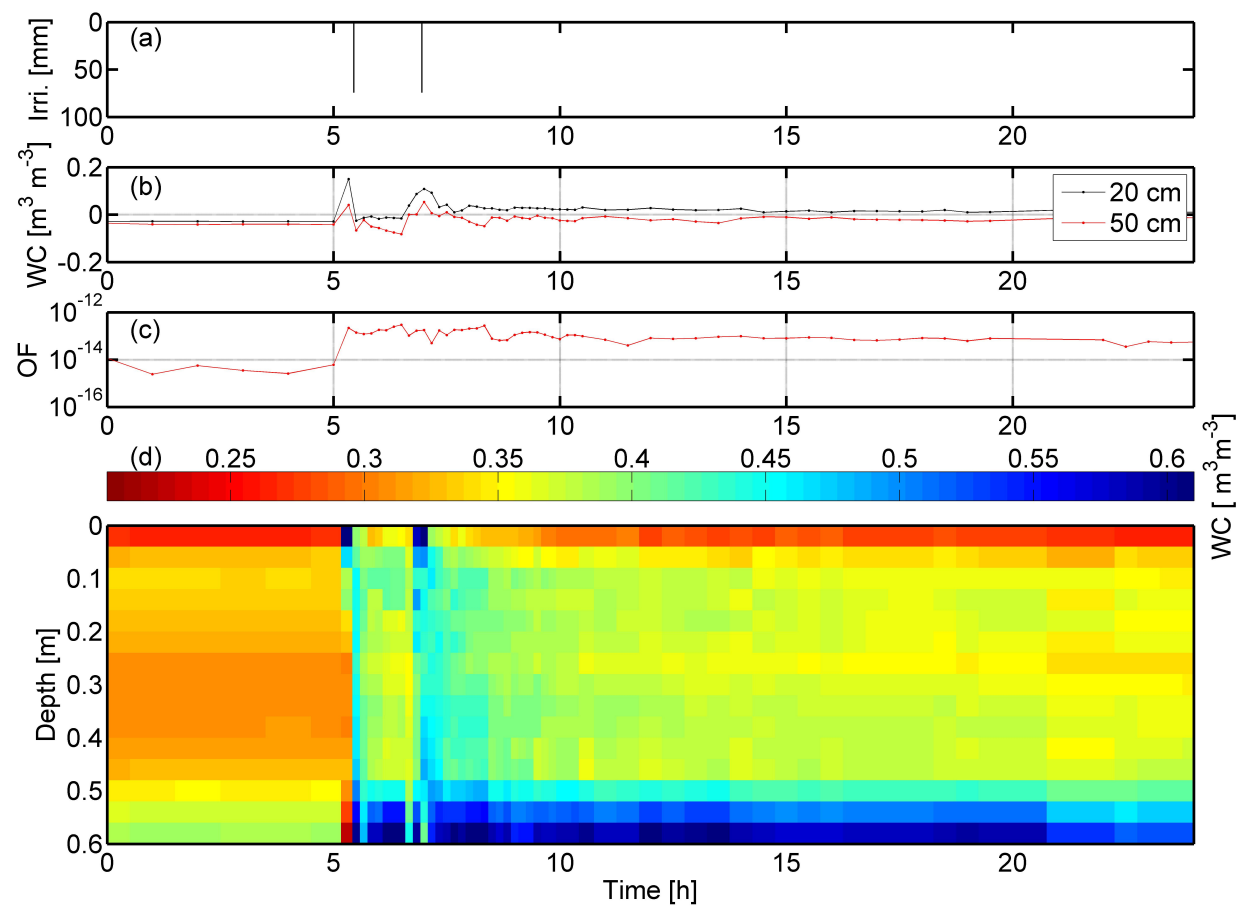

Fig. 9. Inverted soil moisture profiles obtained in an experimental box filled with soil from Rehefeld with (a) the irrigation, (b) the absolute error of inversion compared to measurement with THETA, (c) the objective function (shortened with OF) of the inversion and (d) the inverted profiles to a depth of $0.55 \mathrm{~m}$.

normalized voltage at $5 \mathrm{~ns}$ in Fig. $8 \mathrm{~b}$ and c. The wet peace of wood has no influence in the dry and intermediate case and less effect on the profile in the wet case, although slight heterogeneities in the pore space could be observed and have similar impact on the reflectograms. Inversion yields slightly lower soil moisture at $0.30 \mathrm{~m}$ when compared to the values below and above. The effect of the iron block and the other objects agree with the expected behaviour.

It is important to stress that an ideal conductor in the integration volume has the same influence on the reflectogram and the inverted moisture profiles as a convergent probe geometry (compare Fig. 7a and Fig. 8a). Both lead to a strong decrease in the amplitude of the reflectogram. Fortunately, gravel, boulder blocks and other solid objects of low electric conductivity and low permittivity seem to be not as critical as was expected. Their effect on the reflectogram is rather small.

\subsection{Experiment 5: measurement of soil moisture in disturbed soil}

The applicability of TDR in soils of high clay content is generally hampered because of relaxation phenomena and high energy losses along the transmission line (Chen et al., 2007; Kupfer et al., 2007). As the soil at the field sites contains about $16 \%$ clay, we performed irrigation experiments using field soil material (Table 2) in the box described in experi- ment 1 with the wooden template to guarantee an ideal probe geometry. The plate at the base was perforated to allow for exfiltration of irrigated water. To set up the experiment, we filled the box to $50 \%$ with disturbed soil material from the field site, installed the SUSU 3 probe in the template, installed two THETA probes at depths of $0.25 \mathrm{~m}$ and $0.50 \mathrm{~m}$ and then filled the remaining volume. Additionally, we installed a $0.30 \mathrm{~m}$ uncoated TDR probe of type CS610 (Campbell Scientific Inc.) vertically from the top into the box to measure the bulk electrical conductivity. After filling and probe installation, the soil material was compacted to avoid air gaps between the rods and the surrounding soil. The spinup time of the experiment was two months with an irrigation amount of $741 \mathrm{~m}^{-2}$ every fourth day to achieve stable initial conditions. The actual irrigation experiment lasted $10 \mathrm{~h}$, with a temporal sampling interval of the soil moisture data before irrigation of $20 \mathrm{~min}$ and during and after irrigation of $10 \mathrm{~min}$. The soil was irrigated twice $\left(741 \mathrm{~m}^{-2} 4 \mathrm{~min}^{-1}\right)$ using de-ionized water. During the inversion we used the profile information of the previous time step as the initial condition for the following step. Inverted soil moisture profiles obtained with Spatial TDR were compared to measurements with the THETA probes at two different depths.

Figure 9 presents the temporal development of the inverted soil moisture profile during two irrigations of approximately $741 \mathrm{~m}^{-2}$ in a period of $4 \mathrm{~min}$ at $5: 20 \mathrm{~h}$ and $6: 40 \mathrm{~h}$ (a), the absolute error of inverted profile compared to THETA probe 
measurements (b), the objective function of the inversion (c), and the inverted profiles (d). The range of the bulk conductivity measured with a CS610 was $0.04-0.10 \mathrm{dS} \mathrm{m}^{-1}$. The spatial resolution was aggregated to $0.05 \mathrm{~m}$ length for a better comparability with the THETA probe measurements. During inversion, parameter set "exp. 2" (Table 3) was used to characterize the transmission line.

The soil column was relatively dry before the irrigation, with a dry top layer and a slight increase in soil moisture towards the bottom. During the irrigation, the infiltration front reaches a depth of approximately $0.15 \mathrm{~m}$ in the first $10 \mathrm{~min}$, and then the infiltration front reaches the bottom after further $10 \mathrm{~min}$. Exfiltration starts at the bottom of the soil column, with some $101 \mathrm{~m}^{-2}$ leaving the column in the first few minutes after the infiltration front has reached the bottom. In the top layer, up to a depth of $0.10 \mathrm{~m}$, the soil moisture decreases while the lower layers remain saturated for $2 \mathrm{~h}$, after which the second irrigation experiment is initiated. The wetter soil reacts much faster upon irrigation. Drying then starts again, mainly forced by evaporation, and the profile evolves to the initial conditions. First, the soil moisture decreases from $0.56 \mathrm{~m}^{3} \mathrm{~m}^{-3}$ to $0.25 \mathrm{~m}^{3} \mathrm{~m}^{-3}$ in the top layer up to a depth of $0.08 \mathrm{~m}$ within a few hours. The deeper parts have lower rates of drying depending on the depth, and at the end of the experiment the lowest $0.12 \mathrm{~m}$ were still saturated.

The estimated absolute error (Fig. 9b) of the inverted value minus the measured soil moisture with THETA probes in the depths $0.20 \mathrm{~m}$ and $0.50 \mathrm{~m}$ shows that the inverted value slightly underestimates the soil moisture measured with the THETA probes before the irrigation. During the irrigations, the inversion overestimates the soil moisture, and during the drying the soil moisture is underestimated again. Especially during the irrigation phase, the absolute error is larger for the upper probe $\left(0.15 \mathrm{~m}^{3} \mathrm{~m}^{-3}\right)$ than for the lower one $\left(0.05 \mathrm{~m}^{3} \mathrm{~m}^{-3}\right)$. During the drying phase, the soil moisture for the upper probe is slightly overestimated, and for the lower it is underestimated. One explanation for the differences smaller $<0.03 \mathrm{~m}^{3} \mathrm{~m}^{-3}$ between inverted soil moisture and THETA probe measurements during the experiment is small scale heterogeneity in the soil column.

Figure $9 \mathrm{c}$ shows the temporal development of the objective function for the inversions, which is an uncertainty measure for the inverted soil moisture values. A higher value of the objective function implies a high uncertainty of the measurements. The objective function slightly decreases at the beginning of the experiment. It increases with the start of the irrigation. Between the two irrigation events, the objective function remains constant, but increases again after the second irrigation. Finally, the objective function value decreases continuously with decreasing soil moisture content. The increase of the objective function and the large difference to the upper THETA probe during the irrigation indicate that fast soil moisture changes are problematic for the inversion procedure, which applies especially for the upper part of the probe.

\section{Discussion and conclusions}

Different laboratory experiments were carried out to investigate the feasibility of retrieving soil moisture profiles with Spatial TDR technology in glass beads and heterogeneous loamy soils with substantial clay content.

This study shows that deformation of the probe geometry, which can be assumed to be the rule rather than the exception when installing long TDR probes in heterogeneous soils, influence both the reflectogram and the inverted soil moisture profile. In the case of divergent or convergent rods, neither the inductance $L$ nor the capacitance $C_{1}$ can be assumed to be constant along the transmission line. Fortunately, probe deformations leave the average moisture content along the probe almost unchanged. The average value is determined from the pulse travel time between the first and second main reflections in the reflectogram. Their location is not affected by probe deformations, because we used coated probes here. Average soil moisture values obtained with uncoated probes are, however, more sensitive to probe deformations as shown by Bänninger et al. (2008) and Spittlehouse (2000). Spittlehouse observed a reduction of average soil moisture in the divergent case and predicted an underestimation of the sampling volume for the convergent case. Ferré et al. (1998) showed that coated probes have a clearly smaller sampling volume than uncoated probes and that three rod probes have a smaller sample volume than two rod probes. A decreasing/increasing of the sample volume in the case of convergent/divergent probes has thus a smaller effect when using coated three rod probes, which explains the robustness of average soil moisture contents obtained here. A much stronger deformation than has been investigated here could surely have an effect on the average soil moisture values. However, in the present study we just investigated realistic rod deformations that were observed in a related field study (Zehe et al., 2010).

It is also good news that solid objects like gravel, wood or boulder blocks only have a small effect on the inverted soil moisture profiles. They show up as slightly drier regions in the reflectogram. However, when a solid electrical conductor (an iron block) is present, soil moisture in this region is strongly overestimated by the inversion. Similar problems could occur in soils with a high content of iron-rich minerals, as discussed by Robinson et al. (1994) and Van Dam et al. (2002).

Finally, we found that observations with THETA probes and soil moisture values retrieved from the same depths were generally in good accordance both in glass beads and disturbed natural soil from the field site. It has to be considered that during infiltration or withdrawal, the accuracy of the inverted profile decreases. We demonstrated furthermore that Spatial TDR is capable of monitoring fast infiltration and redistribution of irrigation water in soil.

We have analyzed the sources and subsequent impacts of different kind of errors. The biggest problem is certainly the 
bias that is introduced by probe deformations. The suggested measure CA allows assessing whether the probe is convergent or divergent. During the experiments we found positive values in the case of convergent rods. Negative values are observed for parallel and divergent rods. The absolute value of the negative values increases with increasing divergence. Thus, if the reflectogram of a probe shows strongly negative or positive values under different conditions, it is likely that the probe geometry is deformed. In the case of a small negative $\mathrm{CA}$, the function is not able to give clear information about the probe geometry if the probe is parallel or slightly divergently installed. This could lead to a biased inversion with a slight underestimation in the depth. This error source can only be identified by excavation of the probe. In the case of layered soils where the lower part can be systematically drier/wetter than the upper soil, or the case of gradients in salt, clay or organic content, identification of probe deformations using Eq. (12) is not that straight forward. The amplitude coefficient should to be evaluated for different wetness states, if the amplitude coefficient remains unchanged while observed, a probe deformation is likely. We recommend, furthermore, assessment of detailed information on the soil profile and the soil's physical properties within different layers to assist interpretation of the amplitude coefficient.

Future steps should further elaborate on the calibration of transmission line parameters. Especially for soils rich in fine particles, Eq. (10) should be revisited, to check whether the assumption of zero conductivity is reasonable when $C$ drops below $C_{0}$. We think that independent data on the electrical conductivity of the soil will facilitate solving this problem. Furthermore, information about soil chemical properties and mineral content could be used as a pedotransfer function in supplementary work. It might also be necessary to introduce separate parameterization of the $C-G$ relationship in Eq. (10) for strongly different soil horizons.

Acknowledgements. This study was partly funded by the German Ministry of Education and Research (BMBF) as part of RIMAX (Risikomanagement extremer Hochwasserereignisse), by the University of Potsdam, the Potsdam Graduate School, and by the Technische Universität München. It is part of the OPAQUE (operational discharge and flooding predictions in head catchments) project that aims at improving operational flood forecasting in mountainous headwaters (FKZ number 0330713D). Furthermore, we thank Dominik Reusser, Andre Terwei, Silja Hund, Erik Sommerer, Enrico Grams, Heiko Thoss and Niko Bornemann for their valuable help during the field season and laboratory work, again Dominik Reusser and Theresa Blume and three anonymous reviewers for their valuable comments and Peter Senft for permanent observation of the measurement equipment. We furthermore thank Sven Irrgang from Sachsenforst department Bärenfels for supporting field measurements with Spatial TDR.

Edited by: Z. Su

\section{References}

Alharthi, A. and Lange, J.: Soil water saturation - dielectric determination, Water Resour. Res., 23(4), 591-594, 1987.

Bänninger, D., Wunderil, H., Nussberger, M., and Flühler, H.: Inversion of TDR signals - revisited, J. Plant Nutr. Soil Sc., 171, 137-145, 2008.

Becker, R.: Spatial Time Domain Reflectometry for Monitoring Transient Soil Moisture Profiles, Ph. D. thesis, Mitteilungen des Institutes für Wasser und Gewässerentwicklung - Bereich Wasserwirtschaft und Kulturtechnik mit "Theodor-RehbockWasserbaulaboratorium", Heft 228, University of Karlsruhe, Germany, 2004.

Blume, T., Zehe, E., and Bronstert, A.: Use of soil moisture dynamics and patterns at different spatio-temporal scales for the investigation of subsurface flow processes, Hydrol. Earth Syst. Sci., 13, 1215-1233, doi:10.5194/hess-13-1215-2009, 2009.

Cassiani, G., Binley, A., and Ferré, T. P. A.: Unsaturated zone processes, in: Applied Gydrogeophysics, NATO Science Series, IV. Eart and Environmental Sciences Vol. 71, edited by: Verrecken, H., Binley, A., Cassiani, G., Revil, A., and Titov, C., Springer, Dordrecht, 75-88, 2006.

Chaves, J., Neill, C., Germer, S., Neto, S. G., Krusche, A., and Elsenbeer, H.: Land management impacts on runoff sources in small Amazon watersheds, Hydrol. Process., 22, 1766-1775, 2008.

Chen, R., Drnevich, V. P., Yu, X., Nowack, R., and Chen, Y.: Time domain reflectometry surface reflections for dielectric constant in highly conductive soils, J. Geotech. Geoenviron., 133(12), 15791608, 2007.

Dalton, F. N. and Van Genuchten, M. T.: The time-domain reflectometry method for measuring soil water content and salinity, Geoderma, 38, 237-250, 1986.

Dingman, S. L.: Physical Hydrology, 575 pp., McMillan, New York, 1994.

Ferré, T. P. A., Rudolph, D. L., and Kachanoski, R. G.: Spatial averaging of water content by time domain reflectometry: implications for twin rod probes with and without dielectric coatings, Water Resour. Res., 32, 271-279, 1996.

Ferré, T. P. A., Knight, J. H., Rudolph, D. L., and Kachanoski, R. G.:The sample areas of conventional and alternative time domain reflectometry probes, Water Resour. Res., 34, 2971-2979, 1998.

Friedman, S. P.: Statistical mixing model for the apparent dielectric constant of unsaturated porous media, Soil Sci. Soc. Am. J., 61(3), 742-745, 1997.

Gaskin, G. J. and Miller, J. D.: Measurement of soil water content using a simplified impedance measuring technique, J. Agr. Eng. Res., 63(2), 153-159, 1996.

Greco, R.: Soil water content inverse profiling from single TDR waveforms, J. Hydrol., 317, 325-339, 2006.

Greco, R. and Guida, A.: Field measurements of topsoil moisture profiles by vertical TDR probes, J. Hydrol., 348, 442-451, 2008.

Hakansson, G.: Reconstruction of soil moisture profiles, Master's thesis, Royal Institute of Technology, Department of Electromagnetic Theory, Stockholm, 1997.

Heimovaara, T. J., Huisman, J. A., Vrugt, J. A., and Bouten, W.: Obtaining the spatial distribution of water content along a TDR probe using the SCEM-UA Bayesian inverse modelling scheme, Vadose Zone J., 3, 1128-1145, 2004. 
Herkelrath, W. N., Hamburg, S. P., and Murphy, F.: Automatic realtime monitoring of soil moisture in a remote field area with time domain reflectometry, Water Resour. Res., 27(5), 857-864, 1991.

Huebner, C., Schlaeger, S., Becker, R., Scheuermann, A., Brandelik, A., Schädel, W., and Schuhmann, R.: Advanced measurement methods in time domain reflectometry for soil moisture determination, in: Electromagnetic Aquametry, edited by: Kupfer, K., Springer, Berlin, 317-347, 2005.

Huisman, J. A., Lin, C. P., Weihermüller, L., and Vereecken, H.: Accuracy of Bulk Electrical Conducvity Measurements with Time Domain Reflectometry, Vadose Zone J., 7(2), 426-433, 2008.

Jones, S. B., and Or, D.: Frequency domain analysis for extending time domain reflectometry water content measurement in highly saline soils, Soil Sci. Soc. Am. J., 68, 1568-1577, 2004.

Knight, J. H., Ferré, T. P. A, Rudolph, D. L., and Kachanoski, R. G.: A numerical analysis of the effects of coatings and gaps upon relative dielectric permittivity measurement with time domain reflectometry, Water Resour. Res., 33, 1455-1460, 1997.

Kupfer, K., Trinks, E., Wagner, N., and Hübner, C.: TDR measurements and simulations in high loosy bentonite materials, Meas. Sci. Technol., 18, 1118-1136, 2007.

Landesamt für Umwelt und Geologie: Bodenkonzeptkarte 25 des Freistaates Sachsen, Blatt 5248, Altenberg, 2006.

Lesmes, D. P. and Friedman, S. P.: Relationship between the electrical and hydrogeological properties of rocks and soil, in: Hydrogeophysics, edited by: Robin, Y. and Hubbard, S. S., Water Sci. Technol., Volume 50, Springer, Dordrecht, 2005.

Lin, C .P., Chung, C. C., Huisman, J. A., and Tang, S. H.: Clarification and Calibration of Reflection Coefficient for Electrical Conductivity Measurement by Time Domain Reflectometry, Soil Sci. Soc. Am. J., 72(4), 1033-1040, 2008.

Lin, M. W., Thaduri, J., and Abatan, A. O.: Development of an electrical time domain reflectometry (ETDR) distributed strain sensor, Meas. Sci. Technol., 16, 1495-1505, 2005.

Malicki, M. A., Plagge, R., and Roth, C. H.: Improving the calibration of dielectric TDR soil moisture determination taking into account the solid soil, Eur. J. Soil Sci., 47, 357-366, 1996.

Merz, B., Bárdossy, A., and Schiffler, G. R.: Different methods for modelling the areal infiltration of a grass field under heavy precipitation, Hydrol. Process., 16, 1383-1402, 2002.

Moret, D., Arrúe, J. L., López, M. V., and Gracia. R.: A new TDR waveform analysis approach for soil moisture profiling using a single probe, J. Hydrol., 321, 163-172, 2006.

Moret-Fernández, D., Lera, F., Arrúe, J. L., and López, M. V.: Measurement of soil bulk electrical conducctvity using partially coated TDR probes, Vadose Zone J., 8, 594-600, 2009.

Nichol, C., Beckie, R., and Smith, L.: Evaluation of uncoated and coated time domain reflectometry probes for high electrical conductivity systems, Soil Sci. Soc. Am. J., 66, 1454-1465, 2002.

Oswald, B.: Full wave solution of inverse electromagnetic problems, Ph.D. thesis Swiss Federal Institute of Technology, Zürich, 2000 .

Oswald, B., Benedickter, H. R., Bächtold, W., and Flühler, H.: Spatially resolved water content profiles from inverted time domain reflectometry signals, Water Resour. Res., 39(12), 1357, 2003.
Paasche, H., Tronicke, J., Holliger, K., Green, A. G., and Maurer, H.: Integration of diversephysical-property models: Subsurface zonation and petrophysical parameter estimation based on fuzzy c-means cluster analyses, Geophysics, 71, H33-H44, 2006.

Rejiba, F., Cosenza, P., Camerlynck, C., and Tabbagh, A.: Three-dimensional transient electro-magnetic modeling for investigating the spatial sensitivity of time domain reflectometry measurements, Water Resour. Res., 41, W09411, doi:10.1029/2004WR003505, 2005.

Robinson, D. A., Bell, J. P., and Batchelor, C. H.: Influence of iron minerals on the determination of soil water content using dielectric techniques, J. Hydrol., 161, 169-180, 1994.

Robinson, D. A., Jones, S. B., Wraith, J. M., Or, D., and Friedman, S. P.: A Review of Advances in Dielectric and Electrical Conductivity Measurement in Soils, Vadose Zone J., 2, 444-475, 2003.

Roth, C. H., Malicki, M. A., and Plagge, R.: Empirical evaluation of the relationship between soil dielectric constant and volumetric water content and the basis for calibrating soil moisture measurements by TDR, J. Soil Sci., 43, 1-13, 1992.

Schädel, W.: Schritte zur Verbesserung der Hochwasserwarnung mittels Online-Bodenfeuchtemessungen, Ph.D. thesis, Institute of Water and River Basin Management, Univ. of Karlsruhe, Germany, 2006.

Scheuermann, A., Huebner, C., Schlaeger, S., Wagner, N., Becker, R., and Bieberstein, A.: Spatial-Time Domain Reflectometry and its application for the measurement of water content distributions along flat ribbon cables in a full scale levee model, Water Resour. Res., 45, W00D24, doi:10.1029/2008WR007073, 2009.

Schlaeger, S.: A fast TDR-inversion technique for the reconstruction of spatial soil moisture content, Hydrol. Earth Syst. Sci., 9, 481-492, doi:10.5194/hess-9-481-2005, 2005.

Spittlehouse, D. L.: Using time domain reflectometry in stony forest soil, Can. J. Soil Sci., 80(1), 3-11, 2000.

Starr, J. L. and Timlin, D. J.: Using high-resolution soil moisture data to assess soil water dynamics in the vadose zone, Vadose Zone J., 3, 926-935, 2004.

Topp, G. C., Davis, J. L., and Annan, A. P.: Electromagnetic determination of soil water content: measurement in coaxial transmission lines, Water Resour. Res., 16, 574-582, 1980.

Topp, G. C. and Davis, J. L.: Electromagnetic determination of soil water content using TDR: applications to wetting fronts and steep gradients, Soil Sci. Soc. Am. J., 46, 672-678, 1982.

Van Dam, R. L., Schlager, W., Dekkers, M. J., and Huisman, J. A.: Iron oxides as a cause of GPR reflections, Geophysics, 67(2), 536-545, 2002.

Zehe, E. and Blöschl, G.: Predictability of hydrologic response at the plot and catchment scales: Role of initial conditions, Water Resour. Res., 40, W10202, doi:10.1029/2003WR002869, 2004.

Zehe, E., Elsenbeer, H., Lindenmaier, F., Schulz, K., and Blöschl, G.: Patterns of predictability in hydrological threshold systems, Water Resour. Res., 43, W07434, doi:10.1029/2006WR005589, 2007.

Zehe, E., Graeff, T., Morgner, M., Bauer, A., and Bronstert, A.: Plot and field scale soil moisture dynamics and subsurface wetness control on runoff generation in a headwater in the Ore Mountains, Hydrol. Earth Syst. Sci., 14, 873-889, doi:10.5194/hess14-873-2010, 2010. 